\title{
Competitive Optimal On-Line Leasing
}

\author{
R. El-Yaniv, ${ }^{1}$ R. Kaniel, ${ }^{2}$ and N. Linial $^{3}$
}

\begin{abstract}
Consider an on-line player who needs some equipment (e.g., a computer) for an initially unknown number of periods. At the start of each period it is determined whether the player will need the equipment during the current period and the player has two options: to pay a leasing fee $c$ and rent the equipment for the period, or to buy it for a larger amount $P$. The total cost incurred by the player is the sum of all leasing fees and perhaps one purchase.

The above problem, called the leasing problem (in computer science folklore it is known as the ski-rental problem), has received considerable attention in the economic literature. Using the competitive ratio as a performance measure this paper is concerned with determining the optimal competitive on-line policy for the leasing problem.

For the simplest version of the leasing problem (as described above) it is known and readily derived that the optimal deterministic competitive performance is achieved by leasing for the first $k-1$ times and then buying, where $k=P / c$. This strategy pays at most $2-1 / k$ times the optimal off-line cost.

When considering alternative financial transactions one must consider their net present value. Thus, accounting for the interest rate is an essential feature of any reasonable financial model. In this paper we determine both deterministic and randomized optimal on-line leasing strategies while accounting for the interest rate factor.

It is shown here, for the leasing problem, that the interest rate factor reduces the uncertainty involved. We find that the optimal deterministic competitive ratio is $1+(1+i)(1-1 / k)(1-k(i / 1+i))$, a decreasing function of the interest $i$ (for all reasonable values of $i$ ). For some applications, realistic values of interest rates result in relatively low competitive ratios. By using randomization the on-line player can further boost up the performance. In particular, against an oblivious adversary the on-line player can attain a strictly smaller competitive ratio of $2-\left((k /(k-1))^{\gamma}-2\right) /\left((k /(k-1))^{\gamma}-1\right)$ where $\gamma=\ln (1-k(1-1 /(1+i))) / \ln (1 /(1+i))$. Here again, this competitive ratio strictly decreases with $i$.

We also study the leasing problem against a distributional adversary called "Nature." This adversary chooses the probability distribution of the number of leasing periods and announces this distribution before the on-line player chooses a strategy. Although at the outset this adversary appears to be weaker than the oblivious adversary, it is shown that the optimal competitive ratio against Nature equals the optimal ratio against the oblivious adversary.
\end{abstract}

Key Words. Leasing, Lease-or-buy problem, On-line algorithms, Competitive analysis, Equipment rental, Ski rental.

1. Introduction. In recent years competitive analysis (to be defined) has been gaining recognition for being a complementary approach in the analysis of algorithmic decision making under uncertainty. In this approach one assumes that events are generated by an adversary and the performance of an on-line strategy, in which the decision maker has no knowledge of future events, is compared with that of a clairvoyant strategy that has knowledge of the entire future and operates optimally.

\footnotetext{
${ }^{1}$ Computer Science Department, Technion - Israel Institute of Technology, Haifa 32000, Israel. rani@cs.technion.ac.il.

2 The Wharton Business School, University of Pennsylvania, Philadelphia, PA 19104, USA. kanie173@wharton.upenn.edu.

${ }^{3}$ Institute of Computer Science, The Hebrew University, Jerusalem 91904, Israel. nati@ cs.huji.ac.il.
}

Received October 8, 1997; revised February 8, 1998. Communicated by M.-Y. Kao, A. S. Kyle, and P. Lakner. 
The success of most financial decisions depends on future events of which decision makers have only partial and typically, very scarce knowledge. Despite that, financial agents and institutions have to make periodical decision with irreversible consequences. Thus the nature of most financial problems is inherently on-line.

In the traditional approach to the analysis of financial strategies, sometimes called distributional or average case analysis, one assumes that future events are distributed according to some known or unknown probability distribution and tries to achieve good average case performance with respect to these stochastic assumptions. This approach can be criticized from both technical and conceptual perspectives. Technically, for many real-life problems, an adequate stochastic model is extremely difficult or costly to devise. Sometimes, even if an adequate stochastic model can be found it might be very hard to characterize or compute the optimal strategy analytically. From a conceptual point of view, a strategy that is good on average is not necessarily the best strategy. In many occasions it may be more advantageous to secure some minimal profit (or maximal loss).

1.1. Competitive Analysis. Consider a cost minimization problem $\mathcal{P}$ consisting of a set $\mathcal{I}$ of inputs and a cost function $C$. Associated with each input $I \in \mathcal{I}$ is a set of feasible outputs $F(I)$. For each input $I$ and a feasible output $O \in F(I)$, the cost associated with $I$ and $O$ is $C(I, O) \in \mathbb{R}^{+}$. Let ALG be any algorithm for $\mathcal{P}$. Denote by ALG $[I]$ a feasible output produced by ALG given the input $I$. The cost incurred by ALG is denoted $\operatorname{ALG}(I)=C(I, \operatorname{ALG}[I])$. In general, in an on-line problem $\mathcal{P}$ each input $I$ is a finite sequence $I=i_{1}, i_{2}, \ldots, i_{n}$ and a corresponding feasible output is a finite sequence $O=o_{1}, o_{2}, \ldots, o_{n}$. An on-line algorithm must produce a feasible output in stages such that at the $j$ th stage the algorithm is presented with the $j$ th component of the input $i_{j}$ and must produce the $j$ th component of (a feasible) output $o_{j}$ before the rest of the input is made known. Denote by OPT an optimal off-line algorithm. That is, for each input $I$,

$$
\operatorname{OPT}(I)=\min _{O \in F(I)} C(I, O) .
$$

An on-line algorithm ALG is c-competitive (or "attains a competitive ratio $c$ ") if there exists a constant $\alpha$ such that, for each input $I$,

$$
\operatorname{ALG}(I) \leq c \cdot \operatorname{OPT}(I)+\alpha .
$$

The algorithm ALG is strictly $c$-competitive if it is $c$-competitive with $\alpha=0$. In this paper we are only concerned with strict competitive ratios. The smallest $c$ such that ALG is $c$-competitive is called ALG's competitive ratio. Thus, a $c$-competitive algorithm is guaranteed to incur a cost no larger than $c$ times the smallest possible cost in hindsight for each input sequence.

As can be seen in the above definition, the competitive ratio is a worst case performance measure and therefore it is sometimes convenient to view an on-line problem as a twoperson (zero-sum) game between an adversary and the on-line player. In this game the on-line player chooses an on-line algorithm and makes it known to the adversary. Then the adversary chooses an input sequence. The payoff to the adversary is the performance ratio, optimal off-line cost to on-line cost.

In general, as it is in two-person zero-sum games, randomization is required to obtain optimal (expected) competitive performance (see, e.g., [10]). The extension of the 
competitive ratio measure to randomized on-line algorithms is straightforward ${ }^{4}$ under the assumption that the adversary that generates the input sequence is oblivious to the random choices made by the on-line player. Such an oblivious adversary generates the input sequence in advance, given the probability distributions used by the chosen on-line algorithm. ${ }^{5}$ Specifically, the definition of the randomized competitive ratio with respect to an oblivious adversary is the same as (1) with $E[\operatorname{ALG}(I)]$ replacing $\operatorname{ALG}(I)$ where $E[\cdot]$ is the expectation with respect to the random choices made by ALG.

The use of the competitive ratio for the evaluation of on-line algorithms is called competitive analysis. ${ }^{6}$ Competitive analysis was first used by computer scientists in the 70s in connection with approximation algorithms for NP-hard problems (see, e.g., [21], [24], [25], and [40]) and was explicitly formulated in the 1980s in the seminal work of Sleator and Tarjan [38] on list accessing and paging algorithms. Since then, competitive analysis has been extensively used to analyze and design on-line algorithms for many on-line optimization problems related to computer systems.

Competitive analysis appears to be particularly attractive with regard to financial transactions. A major advantage of this approach over the traditional average-case measure is that the need to construct a probabilistic model is circumvented. This advantage is exemplified in cases where one is unable to specify precisely the relevant underlying distribution of a stochastic model; in such cases the distributional approach might be of little use. Another important feature of the competitive ratio is that it is a relative performance measure. In many situations, economic and financial agents would prefer to compare their performance relative to their peers rather than to maximize their utility in some absolute sense. This type of relative utility function is coined in the finance literature as Keeping Up with the Joneses utility functions (see, for example, [1] or [20]).

Nevertheless, in cases where financial players do possess accurate probabilistic models for input sequences, it would be a waste to ignore this knowledge (which is precisely what the pure competitive ratio does). Indeed, competitive algorithms are often criticized of being overly risk-averse. Recently, the competitive analysis framework has been generalized by al-Binali [7] to include a flexible risk management mechanism. This means that a forecast — in particular, a (partial) probabilistic input model—can be included in an optimization that yields algorithms that perform well given that the forecast comes true but still, even if the forecast fails, the loss remains bounded within a prespecified tolerance.

1.2. The Leasing Problem. In this paper we consider a fundamental economic on-line decision problem called the leasing or lease-or-buy problem. ${ }^{7}$ In this problem an on-line player needs some equipment (e.g., a car, musical instrument) for an initially unknown number of periods (e.g., days, months). At the start of each period it is determined whether or not the equipment will be needed for the current period and the player must choose one of two choices: to lease the equipment for a leasing fee $c$, or to buy it for a larger

\footnotetext{
${ }^{4}$ See [9] for a game theoretic formulation of the corresponding two-person zero-sum game.

${ }^{5}$ Other kinds of adversaries are of adaptive type (see [6]). In this paper we only consider oblivious adversaries.

6 The term "competitive ratio" was coined by Karlin et al. [29].

7 This problem is known in computer science folklore as the "ski-rental" problem. Its simplest variant was first presented by Rudolph as an example of an on-line problem and competitive analysis [36].
} 
amount $P$. Once the player has purchased the equipment, he no longer has to pay leasing fees. The total cost incurred by the player is the sum of all costs incurred (including all leasing fees and perhaps one purchase). The player is interested in minimizing his total cost. Whether the equipment is needed or not for a period is made known on-line at the start of the period. ${ }^{8}$

1.3. Contextual Background and Our Results. Several studies have looked at financial and economic problems from the perspective of competitive analysis (and other related approaches). In particular, the following problems have been considered: portfolio selection [15], [23], [35], [13], [14], [16], [34], [22], [8], search and one-way trading [17], [7], equipment replacement (including mortgage refinancing) [18], [5], stock speculation and gambling (multi-armed bandit) [3], [4] and inventory management [30].

The literature of finance has devoted considerable attention to the analysis of asset leasing contracts. Many of the studies were primarily concerned with the effects and implications of the tax code on the lease versus buy decision (see, e.g., [11], [19], [26], [33], [32], and [37]). For example, one rational for the existence of leasing contracts is to reduce tax payments. If the lessor and the lessee face different effective marginal tax rates, leasing can reduce the total tax bill. The existence of taxes also raises the issue of what is the appropriate discount rate one should use when valuing a lease contract. The papers [37], [33], [26] construct valuation formulas which adjust for taxes. ${ }^{9}$

Although tax-related incentives to lease or buy play an important role in explaining the existence of lease contracts and in valuing leases, there are other considerations. Smith and Wakeman [39] analyze various determinants of corporate leasing policy. Some of these determinants are: What is the expected period of use relative to the useful life of the asset, comparative advantage in asset disposal, managerial compensation related issues, and specialization in risk-bearing issues. They also demonstrate that the incentives they identify can help explain some of the contractual clauses which are used in lease contracts.

Our main concern in this paper is different from the issues addressed in the related finance literature mentioned above. We are interested in determining how does one make the lease-or-buy decision in an environment were the lessee is uncertain for how long he will actually need the underlying asset. One paper that does account for uncertainty with regard to the length of time that the asset is required for is that by McConnell and Schallheim [31]. They use an option pricing framework to value operating leases. The standard operating lease can be cancelled by the lessee at any time. Thus, McConnell and Schallheim view an operating lease for $n$ periods as a compound option. The last lease payment purchases the use of the leased asset over the time remaining until the contract expires. Any of the other lease payments can be viewed as a combination of

\footnotetext{
${ }^{8}$ The lease contracts we consider can be viewed as a sequence of short-term leases (see [32] for the distinction between short-term and long-term leases) or equivalently as a perpetual standard operating lease (see [31]).

${ }^{9}$ These papers use an adjusted present value methodology. The basic idea is that the decision maker should discount the after-tax lease payments and depreciation tax shields at an adjusted discount rate $r^{*}$ which is calculated from a conventional formula for weighted average cost of capital (for an explanation on the weighted average cost of capital see, e.g., [12]).
} 
purchasing the use of the asset until the next lease payment is due and an option to pay the next lease payment. ${ }^{10}$

The simplest variant of the leasing problem we consider here has a very simple known solution. It is known in computer science folklore (and can be easily shown) that the optimal deterministic competitive ratio is $2-1 / k$ where $k=P / c$. In Section 2 we "warm up" by giving a few definitions and presenting the analysis of this "folk version."

When considering alternative financial decisions an agent must consider their net present value. That is, accounting for the market interest rate is an essential feature of any reasonable financial model. In this paper we introduce the nominal interest rate in the market into the model. Although this is only one step toward a more realistic solution of the problem, the introduction of this parameter considerably complicates the analysis and also introduces some new issues that do not exist in the simple problem with no interest rate. For example, in the leasing game with zero interest rate it does not matter if the equipment is used by the lessee in contiguous or noncontiguous periods. In contrast, the total cost incurred by the player may change drastically when gaps of nonusage periods are inserted. Of course, in practice there may be contiguous periods of usage that are separated by short or long breaks. One interesting feature that we discover is that the introduction of an interest rate diminishes the uncertainty involved in (financial) decision making. We determine both deterministic and randomized optimal on-line leasing strategies while accounting for the interest rate factor. Both these ratios are monotone decreasing with the interest rate. Let $i>0$ be the nominal interest rate in the market. This means that the present discount value of one unit of money payable in exactly one time period is the amount, which if invested at the rate of return $i$, would grow to exactly one unit of money at the end of the period. That is, the present discount value is exactly $1 /(1+i)$ units of money. Practical reasoning suggests (see Section 3 ) that only values of $i$ that satisfy $i /(1+i)<1 / k$ make sense. For all such sensible values of $i$ we prove in Section 3 that the optimal deterministic competitive ratio is $1+(1+i)(1-1 / k)(1-k(i / 1+i))$, which strictly improves with the interest rate. This can be interpreted as a reduction of the uncertainty (as a function of the interest rate). For example, consider the following instance of the "ski-rental" problem. Assume a person who has, each year, an opportunity to take a 4 day ski vacation. At some ski resorts it is possible to rent the ski equipment for the entire 4 day vacation for about $\$ 110$. On the other hand, it is possible to buy reasonable quality ski equipment for $\$ 600$. This results in $k=5.45$ and the optimal deterministic competitive ratio is 1.82 . Now assume an annual interest rate of $0.065 .{ }^{11}$ This results in a competitive ratio of about 1.57 .

In Section 3.3 we show that by randomizing the on-line player can improve the competitive ratio (against an oblivious adversary). Our analysis, in the case with no interest rate against an oblivious adversary, can be viewed as a discrete version of a result by Karlin et al. [28], in which they study the spin-block problem in a continuous model. In particular, we show that the optimal randomized competitive ratio (against an

\footnotetext{
${ }^{10}$ See [31] for a brief description of the various provisions that can be written into lease contracts.

11 At the time of writing the U.S. T-bill is at $6.62 \%$.
} 
oblivious adversary) is strictly smaller than the deterministic one for all $k$ and is precisely

$$
2-\frac{(k /(k-1))^{k}-2}{(k /(k-1))^{k}-1}<2-\frac{e-2}{e-1} \approx 1.58 .
$$

We then refine the analysis and determine the optimal randomized solution for the case with interest rates. The optimal competitive ratio in this case is

$$
2-\frac{(k /(k-1))^{\gamma}-2}{(k /(k-1))^{\gamma}-1} \quad \text { where } \quad \gamma=\frac{\ln (1-k(1-1 /(1+i)))}{\ln (1 /(1+i))} .
$$

As in the case $i=0$, it is shown that randomization helps to improve the competitive ratio and that the optimal competitive ratio strictly decreases with $i$ for all feasible values of $i$. For example, returning to the above ski-rental instance, with randomization (and interest rate of 0.065 ) we can attain a competitive ratio of 1.37 .

As mentioned above, when interest rates are introduced the total cost incurred by the player may vary drastically if gaps of time periods with no leasing are introduced in between leasing periods. This could potentially increase the competitive ratio. In Section 4 we show that our results hold whether or not the the leasing periods are contiguous.

In Section 5 we analyze the leasing problem against a distributional adversary called "Nature"; this is an adversary that chooses the probability distribution that governs the number of leasing periods and announces this distribution before the on-line player chooses his strategy. Although at the outset this adversary appears to be weaker than the oblivious adversary, it is shown that the competitive ratio against Nature is identical to the ratio against the oblivious adversary.

2. The Folk Version-“the Ski-Rental Problem." We make use of the following notation. For any strategy $X$ for this problem, let $V_{X}(n)$ be the present value of the total cost spent by $X$ if the equipment is used for $n$ periods (in this section, since interest rates are not involved, for all $n$ the present value is simply the total cost). Let OPT be the optimal off-line strategy. We are interested in identifying the optimal on-line leasing strategy and determining the best attainable competitive ratio for this problem; that is, to determine the quantity $\min _{X} \max _{n} V_{X}(n) / V_{\mathrm{OPT}}(n)$.

As a "warm up," we first consider the well-known "folk" variant of the leasing problem called the ski-rental problem. This variant is specified by the following parameters:

- A purchase price, $P$.

- A leasing price, $c$. It is convenient to assume that $P=k c$ for some integer $k>1$. Note that for $k=1$ the problem is trivial since the player buys on the first period.

The first observation is that $V_{\mathrm{OPT}}(n)=\min \{P, n c\}$. Consider the following deterministic on-line strategy: rent up to $k-1$ times and then buy. Thus if $n \leq k-1$, then always lease. Denote this on-line strategy by $\bar{S}$.

It is not hard to see that $\bar{S}$ is $(2-1 / k)$-competitive: If $n \leq k-1$ the on-line and off-line players incur the same cost. On the other hand, if $n>k-1$, then the on-line player pays $(k-1) c+P$ and the off-line player pays $P$. Therefore $\bar{S}$ attains a competitive 
ratio of

$$
\frac{(k-1) c+P}{P}=\frac{(k-1) c+k c}{k c}=2-\frac{1}{k} .
$$

Further, it is not hard to show that $\bar{S}$ is an optimal deterministic strategy. First, notice that any deterministic leasing strategy is a threshold-type strategy, specified by one positive integer $t$ such that the on-line player leases $t-1$ times and then buys. Let $S(t)$ be such a strategy with $t \neq k$. Clearly, the best option for the adversary is to choose $n=t$. Consider the two cases:

(i) $t<k$ : then $S(t)$ attains a competitive ratio of $((t-1) c+P) / t c=1+(k-1) / t \geq$ 2.

(ii) $t>k$ : now $S(t)$ attains a competitive ratio $((t-1) c+P) / P>((k-1) c+P) / P=$ $2-1 / k$.

REMARK 1. For an arbitrary $k$ (not necessarily an integer) it can be shown that the competitive ratio of the optimal on-line strategy is

$$
\min \left\{1+\frac{k-1}{\lfloor k\rfloor}, 1+\frac{\lfloor k\rfloor}{k}\right\} .
$$

This competitive ratio can be obtained by a strategy similar to $S(t)$.

3. Leasing in a Market with Interest Rate. As before, let $P$ and $c<P$ be the purchase price and the per-period leasing price, respectively. In addition, let $i$ be the nominal interest rate in the market. Without loss of generality we assume that $c / P>$ $i /(1+i)$. This is a reasonable assumption for any practical use because the purchase price of the equipment must be less than the present discount value of the alternative of always leasing $\left(\sum_{j=0}^{\infty}\left(c /(1+i)^{j}\right)>P\right)$. Otherwise, the on-line player can attain a competitive ratio of 1 by simply never purchasing the equipment.

Throughout this section we make the simplifying assumption that the player needs the equipment throughout $n$ contiguous time periods. (Later, in Section 4, we prove that this assumption can be taken without loss of generality.)

3.1. Optimal Off-Line Strategy. Clearly, for any $n$ the optimal off-line decisions are made according to one of the following two rules: (i) always lease; (ii) rent $t$ times and then buy, where $0 \leq t \leq n-1$.

Set $\beta=1 /(1+i)$. For $i>0$ the present discount value of $n$ rentals is

$$
\sum_{j=0}^{n-1} \frac{c}{(1+i)^{j}}=c \sum_{j=0}^{n-1} \beta^{j}=c \frac{1-\beta^{n}}{1-\beta} .
$$

The present value of $t$ rentals followed by a purchase is

$$
f(t)=\beta^{t}\left(P-\frac{c}{1-\beta}\right)+\frac{c}{1-\beta} .
$$


Using the assumption that $c / P>i /(1+i)$ we obtain (as in the "folk" version) that OPT will never purchase the equipment after leasing it for some time.

Let $n^{*}$ be the number of rentals whose total present value is $P$. In other words, $n^{*}$ is the root of $c\left(\left(1-\beta^{n}\right) /(1-\beta)\right)=P$. That is

$$
\beta^{n^{*}}=1-\frac{P(1-\beta)}{c} .
$$

Therefore,

$$
n^{*}=\frac{\ln (1-k(1-\beta))}{\ln \beta}=\frac{\ln (1-k(i / 1+i))}{\ln (1 /(1+i))} .
$$

REMARK 2. By applying straightforward calculus it can be shown that $n^{*}$ is a continuous function of $i(i \geq 0)$ and that $\lim _{i \rightarrow 0} n^{*}=k$.

It follows that, for any $n$, the optimal off-line cost, $V_{\mathrm{OPT}}(n)$, is given by

$$
V_{\mathrm{OPT}}(n)= \begin{cases}c \frac{1-\beta^{n}}{1-\beta}, & n<n^{*}, \\ P, & n \geq n^{*} .\end{cases}
$$

3.2. Optimal On-Line Deterministic Strategy. In what follows we make the simplifying assumption that $n^{*}$ is an integer. ${ }^{12}$

THEOREM 3.1. The optimal competitive ratio obtained when $i>0$ is

$$
1+\frac{1}{\beta}\left(1-\frac{1}{k}\right)(1-k(1-\beta))=1+(1+i)\left(1-\frac{1}{k}\right)\left(1-k \frac{i}{1+i}\right) .
$$

This competitive ratio can be obtained by following on-line strategy $S^{*}$ :

- If $n \leq n^{*}-1$, then always lease.

- Otherwise, lease $n^{*}-1$ periods and then buy.

PROOF. For each $t \geq 1$, let $S(t)$ be the strategy that leases for the first $t-1$ periods and then buys. Thus, $S(t)$ pays

$$
c \cdot \frac{1-\beta^{t-1}}{1-\beta}+P \cdot \beta^{t-1} .
$$

Fix some $t<n^{*}$ and consider the strategy $S(t)$. As in the case with zero interest rate, it is clear that the optimal choice of $n$ by the adversary against $S(t)$ would be exactly $t$. For $n=t$, the cost ratio (on-line/off-line) is

$$
\begin{aligned}
R_{1} & =\frac{c\left(\left(1-\beta^{t-1}\right) /(1-\beta)\right)+P \beta^{t-1}}{c\left(\left(1-\beta^{t}\right) /(1-\beta)\right)} \\
& =\frac{\beta^{t-1}(P-c)+c-P \beta^{t}}{c\left(1-\beta^{t}\right)} .
\end{aligned}
$$

\footnotetext{
12 This assumption is made to facilitate a simpler presentation of the results which can be generalized to an arbitrary $n^{*}$ (see Remark 3).
} 
Note that

$$
\frac{\partial}{\partial t} R_{1}(t)=\frac{\ln \beta \cdot \beta^{t-1}(P-c)(1-\beta)}{c\left(1-\beta^{t}\right)^{2}}<0 .
$$

Therefore, the on-line player will take the maximum possible value of $t$, such that $t<n^{*}$. Having assumed $n^{*}$ to be an integer, $t=n^{*}-1$ and using (2) we obtain

$$
\begin{aligned}
R_{1}\left(n^{*}-1\right) & =\frac{\beta^{n^{*}-2}(P-c)+c-P \beta^{n^{*}-1}}{c\left(1-\beta^{n^{*}-1}\right)} \\
& =1+\frac{(k-1) \beta^{n^{*}-2}(1-\beta)}{1-\beta^{n^{*}-1}} \\
& =1+\frac{1}{\beta} \frac{(k-1)(1-\beta)(1-k(1-\beta))}{\beta-(1-k(1-\beta))} \\
& =1+\frac{1}{\beta}(1-k(1-\beta)) .
\end{aligned}
$$

Next we consider strategies $S(t)$ with $t \geq n^{*}$. There are two mutually exclusive cases. If the choice of $n$ is such that $n<t$, then for $n<n^{*}$ the on-line and off-line costs are equal and for any choice of $n$ with $n^{*} \leq n<t$, the on-line strategy will always lease, incurring a cost of $c\left(\left(1-\beta^{n}\right) /(1-\beta)\right)$. OPT, on the other hand, will buy and pay $P$. Without loss of generality, assume that $n=t-1$. Thus, for this case the best attainable cost ratio is

$$
R_{2}=\frac{c}{P} \cdot \frac{1-\beta^{t-1}}{1-\beta} .
$$

In the second case, the adversary chooses $n \geq t$. Without loss of generality assume that $n=t$ (both the on-line and the off-line players cease all activities by the $t$ th period). The off-line cost is $P$ and the on-line cost is $c\left(\left(1-\beta^{t-1}\right) /(1-\beta)\right)+P \beta^{t-1}$. Thus, the best attainable ratio for this case is

$$
R_{3}=\frac{c}{P} \cdot \frac{1-\beta^{t-1}}{1-\beta}+\beta^{t-1} .
$$

Clearly, $R_{3}>R_{2}$. Therefore, the adversary will choose $n=t$, enforcing the the larger ratio, $R_{3}$. Clearly,

$$
\frac{\partial}{\partial t} R_{3}(t)=\frac{\beta^{t-1} \ln \beta(\beta+c / P-1)}{\beta-1},
$$

which is always positive.

Therefore, for this case, the best attainable ratio is obtained by setting $t=n^{*}$. Thus, using (2) we obtain

$$
\begin{aligned}
R_{3}\left(n^{*}\right) & =\frac{c}{P} \frac{1-\beta^{n^{*}-1}}{1-\beta}+\beta^{n^{*}-1} \\
& =\frac{1}{k} \cdot \frac{1-\beta^{n^{*}}}{1-\beta}+\frac{1}{\beta}\left(1-\frac{1}{k}\right) \beta^{n^{*}}
\end{aligned}
$$




$$
\begin{aligned}
& =1+\frac{1}{\beta}\left(1-\frac{1}{k}\right) \beta^{n^{*}} \\
& =1+\frac{1}{\beta}\left(1-\frac{1}{k}\right)(1-k(1-\beta)) .
\end{aligned}
$$

It is evident that $R_{1}\left(n^{*}-1\right)>R_{3}\left(n^{*}\right)$. Hence the on-line player chooses $t=n^{*}$ (recall that $n^{*}$ is an integer) and the best attainable competitive ratio is

$$
\begin{aligned}
R_{3}\left(n^{*}\right) & =1+\frac{1}{\beta}\left(1-\frac{1}{k}\right)(1-k(1-\beta)) \\
& =1+(1+i)\left(1-\frac{1}{k}\right)\left(1-k \frac{i}{1+i}\right),
\end{aligned}
$$

which is achieved by the optimal strategy $S^{*}$ (i.e., if $n \leq n^{*}-1$, then always lease; otherwise, lease $n^{*}-1$ periods and then buy).

REMARK 3. It can be shown that for an arbitrary $n^{*}$ the competitive ratio attained is $\min \left\{R_{3}\left(\left\lceil n^{*}\right\rceil\right), R_{1}\left(\left\lfloor n^{*}\right\rfloor\right)\right\}$ in which case the competitive ratio can be obtained by a strategy similar to $S^{*}$.

We now examine the optimal competitive ratio as a function of $i$ and of $k$. Observe the following two properties: (i) When $i \geq 0$ the optimal competitive ratio is strictly decreasing with $i$ and strictly increasing with $\beta$. (ii) $1<k<1 /(1-\beta)$ and it is easy to show that the optimal competitive ratio increases in the interval $(1, \sqrt{1 /(1-\beta)}]$, decreases in the interval $[\sqrt{1 /(1-\beta)}, 1 /(1-\beta))$, and has a maximum at $k=1 / \sqrt{1-\beta}$.

3.3. Optimal On-Line Randomized Leasing Strategy. In this section we show how randomization can boost the performance. We consider a game against an oblivious adversary (see Section 1.1). We first consider the case $i=0$ and then generalize to any (feasible) $i>0$.

3.3.1. Randomization when $i=0$. Assume $i=0$, which corresponds to the "folk" version of Section 2. As mentioned earlier, for every $n$, the optimal off-line cost is $V_{\mathrm{OPT}}(n)=\min \{c n, P\}$. As before, for every $t \geq 1$, let $S(t)$ be the deterministic on-line strategy that leases for the first $t-1$ periods and then buys.

Any randomized leasing strategy can be specified by a probability distribution $\left\{p_{t}\right\}$ over deterministic leasing strategies $S(t)$ as defined above. For each $t$ define $p_{t}$ to be the probability of using the strategy $S(t)$. Let $R=R(t, n, k)$ denote the cost ratio (online/off-line) of the strategy $S(t)$ when the game consists of $n$ periods.

THEOREM 3.2. The optimal randomized competitive ratio against an oblivious adversary is

$$
\frac{1}{1-((k-1) / k)^{k}}=2-\frac{(1+1 /(k-1))^{k}-2}{(1+1 /(k-1))^{k}-1} \text {. }
$$


This ratio is attained by the following randomized strategy:

$$
p_{j}= \begin{cases}\frac{1}{1+k\left((1+1 /(k-1))^{k-1}-1\right)}, & j=1 ; \\ p_{1} \cdot\left(\frac{k}{k-1}\right)^{j-1}, & 1<j \leq k ; \\ 0, & k<j .\end{cases}
$$

The proof of Theorem 3.2 makes use of the following lemmas.

LEMMA 3.1. There exists an optimal on-line randomized leasing strategy having the property that, for every $j>k, p_{j}=0$.

PROOF. We first claim that the lemma holds under the assumption that there exists an optimal on-line strategy whose probability distribution has finite support.

To prove this claim consider such an optimal on-line randomized strategy, $P=\left\{p_{j}\right\}$, and set $M(P)=\max \left\{i \mid p_{i} \neq 0\right\}$. The expected (optimal) competitive ratio is thus $R_{1}\left(\left\{p_{t}\right\}, n\right)=\sum_{t} p_{t} \cdot R(t, n, k)$. Without loss of generality assume that $M(P)>k$. It is easy to see that:

(i) Without loss of generality the adversary considers only $n \leq M(P)$.

(ii) $\forall j>n, R(j, n, k)=R(n+1, n, k)$.

(iii) $\forall h>n \geq k, \forall t \leq n, R(t, h, k)=R(t, n, k)$.

(iv) $\forall n>k, R(n, n, k)>R(n-1, n-1, k)$.

Consider the following probability distribution, $\tilde{P}=\left\{\tilde{p}_{t}\right\}$ :

$$
\tilde{p}_{t}= \begin{cases}p_{t}, & \forall t \leq M(P)-2 \\ p_{M(P)-1}+p_{M(P)}, & t=M(P)-1 ; \\ 0, & t=M(P) .\end{cases}
$$

It follows that $M(\tilde{P})=M(P)-1$.

We now check how the expected competitive ratio changes as we shift from the strategy $\left\{p_{t}\right\}$ to the strategy $\left\{\tilde{p}_{t}\right\}$ :

$$
\begin{aligned}
R_{1}\left(\left\{\tilde{p}_{t}\right\}, M(\tilde{P})\right) & =\sum_{t} \tilde{p}_{t} \cdot R(t, M(\tilde{P}), k)=\sum_{t} \tilde{p}_{t} \cdot R(t, M(P)-1, k) \\
& =\sum_{t=1}^{M(P)-2} p_{t} \cdot R(t, M(P)-1, k)+\tilde{p}_{M(P)-1} \cdot R(M(P)-1, M(P)-1, k) \\
& =\sum_{t=1}^{M(P)-1} p_{t} \cdot R(t, M(P)-1, k)+p_{M(P)} \cdot R(M(P)-1, M(P)-1, k) \\
& <\sum_{t=1}^{M(P)-1} p_{t} \cdot R(t, M(P)-1, k)+p_{M(P)} \cdot R(M(P), M(P), k)
\end{aligned}
$$




$$
=\sum_{t=1}^{M(P)} p_{t} \cdot R(t, M(P), k)=R_{1}\left(\left\{p_{t}\right\}, M(P)\right)
$$

and, for all $n<M(\tilde{P})$,

$$
\begin{aligned}
R_{1}\left(\left\{\tilde{p}_{t}\right\}, n\right) & =\sum_{t} \tilde{p}_{t} \cdot R(t, n, k) \\
& =\sum_{t=1}^{M(P)-2} p_{t} \cdot R(t, n, k)+\tilde{p}_{M(P)-1} \cdot R(M(P)-1, n, k) \\
& =\sum_{t=1}^{M(P)-1} p_{t} \cdot R(t, n, k)+p_{M(P)} \cdot R(M(P)-1, n, k) \\
& =\sum_{t=1}^{M(P)-1} p_{t} \cdot R(t, n, k)+p_{M(P)} \cdot R(M(P), n, k)=R_{1}\left(\left\{p_{t}\right\}, n\right) .
\end{aligned}
$$

Thus, the on-line strategy given by $\left\{\tilde{p}_{t}\right\}$ is at least as good as the strategy given by $\left\{p_{t}\right\}$. Repeated applications of this argument prove the claim.

For the case in which the optimal strategy has infinite support (i.e., there are infinitely many indices $j$ such that $p_{j} \neq 0$ ) we show that, for every $0<\varepsilon<1$, there exists a probability distribution $\tilde{P}(\varepsilon)$ with a finite support such that, for all $n$,

$$
R_{1}\left(\left\{\tilde{p}_{t}\right\}, n\right) \leq \frac{R_{1}\left(\left\{p_{t}\right\}, n\right)}{1-\varepsilon} .
$$

Let $0<\varepsilon<1$ be given and let $N(\varepsilon)$ be an index such that $\sum_{N(\varepsilon) \leq t} p_{t}=\delta \leq \varepsilon$. Define

$$
\tilde{p}_{t}= \begin{cases}\frac{p_{t}}{1-\delta}, & t<N(\varepsilon), \\ 0, & N(\varepsilon) \leq t .\end{cases}
$$

Clearly, for all $n,(1-\delta) R_{1}\left(\left\{\tilde{p}_{t}\right\}, n\right) \leq R_{1}\left(\left\{p_{t}\right\}, n\right)$.

Lemma 3.1 tells us that it is sufficient to consider strategies that give positive weights only to the first $k$ periods. We may thus assume that $n \leq k$ and it follows that for any such choice of $n$ the expected cost ratio is

$$
R(n)=1+\sum_{t \leq n} p_{t} \frac{t+k-n-1}{n} .
$$

Define $\triangle^{(k)} \stackrel{\text { def }}{=}\left\{\left(x_{1}, x_{2}, \ldots, x_{k}\right): \sum_{i=1}^{k} x_{i}=1 ; \forall i, x_{i} \geq 0\right\}$.

LEMMA 3.2. Let $A$ be $a k \times k$ positive upper triangular matrix in which the upper elements increase along columns (i.e., $t^{\prime}<t \leq j \leq k$ implies $\left.a_{t^{\prime}, j}<a_{t, j}\right)$. Let $(p, \lambda)$ be an optimal solution for the linear program

$\min \lambda$

$$
\text { such that }\left\{\begin{aligned}
p \cdot A & \leq \lambda \cdot \overrightarrow{1}, \\
p & \in \Delta^{(k)} .
\end{aligned}\right.
$$

Then $p \cdot A=\lambda \cdot \overrightarrow{1}$. 


$$
\begin{aligned}
& \alpha \leftarrow \max _{t, n}\left|a_{t, n}\right| ; \\
& \underline{1:} \delta \leftarrow \min \left\{\lambda-p \cdot A_{j} \mid p \cdot A_{j}<\lambda\right\} ; \\
& l \leftarrow \max \left\{j \mid p \cdot A_{j-1}<p \cdot A_{j}=\lambda\right\} ; \\
& \text { If } l=-\infty \text { then goto } \underline{2} \text {; } \\
& \text { else \{ } \\
& \varepsilon \leftarrow \min \left\{\frac{\delta}{4 \alpha}, \frac{p_{l}}{2}\right\} ; \\
& \bar{p}_{j} \leftarrow \begin{cases}p_{j}+\varepsilon, & j=l-1 ; \\
p_{j}-\varepsilon, & j=l ; \\
p_{j}, & \text { otherwise }\end{cases} \\
& \text { \} } \\
& \text { If } \bar{p} \cdot A<\lambda \cdot \overrightarrow{1} \quad \text { END; } \\
& \text { else }\{ \\
& p \leftarrow \bar{p} \\
& \text { goto } 1 \text {; } \\
& \text { \} } \\
& \text { 2: } \quad r \leftarrow \max \left\{j \mid p \cdot A_{j}=\lambda\right\} \text {; } \\
& \varepsilon \leftarrow \min \left\{\frac{\delta}{4 \alpha}, \frac{p_{1}}{2}\right\} \text {; } \\
& \bar{p}_{j} \leftarrow \begin{cases}p_{1}-\varepsilon, & j=1 ; \\
p_{j}, & 1<j \leq r ; \\
p_{j}+\varepsilon, & j=r+1 ; \\
p_{j}, & j>r+1 ;\end{cases}
\end{aligned}
$$

Fig. 1. An algorithm that computes a probability distribution $\bar{p} \in \Delta^{(k)}$ for which $\bar{p} \cdot A<\lambda \cdot \overrightarrow{1}$.

ProOF. The idea of the proof is that if there exists $j \leq k$ such that $p \cdot A_{j}<\lambda$, then by a small variation of the probability distribution $p$ to $\bar{p}$ we can reduce the values of $p \cdot A_{i}$ in columns, where $p \cdot A_{i}=\lambda$, while in other columns where $p \cdot A_{i}<\lambda$ the value still stays strictly smaller than $\lambda-\gamma$.

By contradiction assume that there exists $j \leq k$ for which $p \cdot A_{j}<\lambda$. Consider the algorithm of Figure 1 . This algorithm computes a probability distribution $\bar{p} \in \triangle^{(k)}$ for which $\bar{p} \cdot A<\lambda \cdot \overrightarrow{1}$.

After executing the algorithm of Figure $1, \bar{p} \cdot A<\lambda \cdot \overrightarrow{1}$. This is established by considering the following three observations:

(i) After each iteration of stage $1, \bar{p} \cdot A \leq \lambda \cdot \overrightarrow{1}$, and $\bar{p} \cdot A_{l}<p \cdot A_{l}=\lambda$ :

- $m<l-1: \bar{p} \cdot A_{m}=p \cdot A_{m}$.

- $m=l-1: \bar{p} \cdot A_{m}=p \cdot A_{m}+\varepsilon a_{m, m} \leq p \cdot A_{m}+(\delta / 4 \alpha) \alpha<\lambda$.

- $m \geq l: \bar{p} \cdot A_{m}=p \cdot A_{m}+\varepsilon a_{l-1, m}-\varepsilon a_{l, m}<\lambda$. 
(ii) The algorithm terminates: from (i) it is clear that the number of times we execute stage 1 is finite and stage 2 is either not executed at all or executed only once.

(iii) After an execution of stage $2, \bar{p} \cdot A<\lambda \cdot \overrightarrow{1}$ :

- $m \leq r: \bar{p} \cdot A_{m}<p \cdot A_{m}=\lambda$.

- $m \geq r+1: \bar{p} \cdot A_{m} \leq p \cdot A_{m}+\varepsilon a_{r+1, m} \leq p \cdot A_{m}+(\delta / 4 \alpha) \alpha<\lambda$.

This completes the proof of the lemma.

Lemma 3.2 entails the following corollary.

COROLLARY 3.1. There exists an optimal on-line randomized leasing strategy with the property that, for every $j \leq k$, the ratios $R(j)$ are equal.

By Corollary 3.1, in order to identify the optimal randomized strategy it remains to solve the system of equations $R(1)=R(2)=\cdots=R(k)$ for the the $p_{i}$ 's under the constraints $p_{i} \geq 0$ and $\sum_{i} p_{i}=1$.

The proof of the following lemma is easily obtained by substitution.

LEMMA 3.3. For every positive $p_{1}$, the choice $p_{j}=p_{1} \cdot(k /(k-1))^{j-1}, j=2, \ldots, k$, solves the system $R(1)=R(2)=\cdots=R(k)$ and, for all $j, R(j)=1+p_{1}(k-1)$.

PROOF OF THEOREM 3.2. Using Lemma 3.3 together with the constraint that $\left\{p_{i}\right\}$ is a probability distribution we calculate $p_{1}$ which must be the solution of $1-\sum_{i=2}^{k} p_{i}=p_{1}$ :

$$
p_{1}=\frac{1}{1+k\left((1+1 /(k-1))^{k-1}-1\right)} .
$$

Note that since $(1+1 /(k-1))^{k-1}$ is strictly increasing toward $e, p_{1}$ is strictly decreasing.

By substituting this value for $p_{1}$ we calculate the optimal expected competitive ratio which after rearrangement reduces to

$$
\frac{1}{1-((k-1) / k)^{k}}=2-\frac{(1+1 /(k-1))^{k}-2}{(1+1 /(k-1))^{k}-1} .
$$

Notice that the optimal deterministic ratio is always in the interval $\left[\frac{3}{2}, 2\right)$ and the optimal randomized ratio is in $\left[\frac{4}{3}, 1.582\right)$. Further, it can be shown that the optimal randomized competitive ratio is strictly smaller than the optimal deterministic one for every $k$.

3.3.2. Randomization When $i>0$. Here we consider randomization when $i$ is positive and state without a proof the following theorem. ${ }^{13}$

\footnotetext{
13 The proof of Theorem 3.3 can be found in [27].
} 


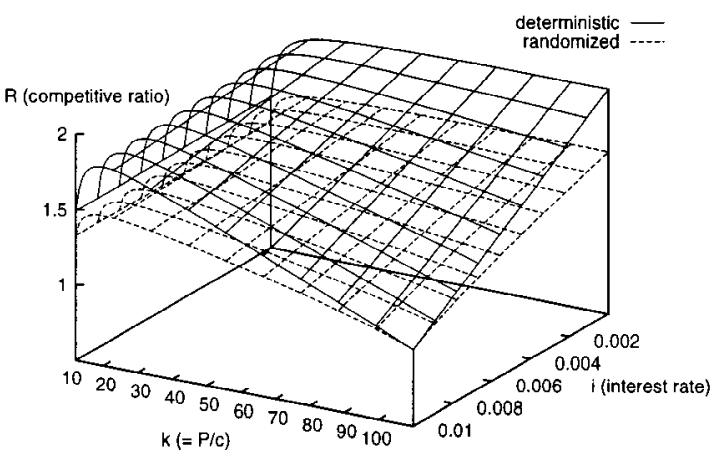

Fig. 2. The optimal on-line randomized competitive ratio is strictly smaller than the optimal deterministic competitive ratio, for all feasible pairs of $k$ and $i$.

THEOREM 3.3. Let $i>0$ be given and set $n^{*}$ to be the number of rentals whose total present value is $P$. Then the optimal competitive ratio against an oblivious adversary is

$$
\frac{1}{1-((k-1) / k)^{n^{*}}}=2-\frac{(k /(k-1))^{n^{*}}-2}{(k /(k-1))^{n^{*}}-1} \text {. }
$$

This optimal competitive ratio can be obtained by the following randomized strategy:

$$
p_{j}= \begin{cases}\frac{1}{k-1} \cdot \frac{(k-1)^{n^{*}}}{k^{n^{*}}-(k-1)^{n^{*}}}, & j=1, \\ p_{1} \cdot\left(\frac{k}{k-1}\right)^{j-1}, & 1<j \leq n^{*}, \\ 0, & n^{*}<j .\end{cases}
$$

We examine how the competitive ratio changes as a function of the interest rate $i$ and of $k$. Clearly:

1. Since $i \geq 0, i \uparrow \Longrightarrow \beta \downarrow \Longrightarrow n^{*} \uparrow \Longrightarrow R \downarrow$.

2. Given that $1<k<1 /(1-\beta)$ (as argued in Section 3 ) it is possible to show that the competitive ratio increases in the interval $(1,1 / \sqrt{1-\beta}]$, decreases in the interval $[1 / \sqrt{1-\beta}, 1 /(1-\beta))$ and obtains a maximum at $k=1 / \sqrt{1-\beta}$.

It can also be shown, that the optimal on-line randomized competitive ratio is strictly smaller than the optimal deterministic competitive ratio, for all feasible pairs of $k$ and $i$ (see Figure 2).

4. Noncontiguous Usage of Equipment. So far we have analyzed the leasing game subject to the assumption that the player uses the equipment for a number of contiguous periods and then never uses it again. Nevertheless, in practice there may be contiguous periods of usage that are separated by short or long breaks. 
Of course, for the leasing game with zero interest rate this contiguous-usage assumption is not required for the analysis as all costs are independent of time. On the other hand, when $i>0$ it is not clear at the outset whether or not the removal of this contiguous-usage assumption will affect the optimal competitive ratio.

Let $U=u_{1}, u_{2}, \ldots, u_{n}$, where the $u_{i}$ 's are period indices, $u_{1}<u_{2}<\cdots<u_{n}$, such that the equipment is needed by the player during each period $u_{i}$. We call $U$ a usage sequence. In each usage sequence $U,|U|=n$ is thus the number of usage periods in the sequence. A usage sequence $U$ is contiguous if $u_{j+1}=u_{j}+1, j=0,1, \ldots$, $n-1$.

Assume that $i>0$. Clearly, since a contiguous usage sequence is a special case of a usage sequence (that may contain gaps of nonusage period segments), the competitive ratio in the noncontiguous usage case can only increase relative to contiguous usage. In this section we prove that in fact it remains the same.

THEOREM 4.1. Let $P, c$, and $i$ be given. Then the competitive ratio in the contiguous case equals the optimal competitive ratio in the noncontiguous case.

Consider the following on-line deterministic strategy $S^{* *}$ : lease the equipment for the first $n^{*}-1$ usage periods and then buy the equipment on the $n^{*}$ usage period. (For a contiguous usage sequence the strategy $S^{* *}$ coincides with the optimal on-line strategy $S^{*}$.)

PROOF OF THEOREM 4.1. We show that $S^{* *}$ ensures a competitive ratio of $1+(1 / \beta)(1-$ $1 / k)(1-k(1-\beta))$ (which is the same as the competitive ratio achieved in the contiguous case).

Before getting into the details we outline the proof. Since the on-line player uses strategy $S^{* *}$ we may assume, without loss of generality, that $|U| \leq n^{*}$ (a longer sequence can only raise the cost for OPT without affecting the on-line cost). We show that for such usage sequences OPT leases the equipment throughout the entire sequence (in the case where $U$ is contiguous with $|U|=n^{*}$, OPT either leases throughout the entire sequence or purchases the equipment on the first period; the price incurred is the same). Based on the fact that OPT leases the equipment throughout the whole sequence we conclude that for any given noncontiguous sequence the adversary can increase the competitive ratio obtained by eliminating all the gaps. Thus, the optimal strategy for the adversary (against $S^{* *}$ ) is to choose a contiguous sequence $U$ with $|U|=n^{*}$.

We now turn to a formal proof of the theorem. With respect to a usage sequence $U=u_{1}, \ldots, u_{n}$ define the following strategies:

- $\operatorname{BUY}(t)$ : lease the equipment until period $u_{t-1}$ and buy it on period $u_{t}$.

- $\operatorname{BUY}(\infty)$ : always lease.

In what follows we use $C(\cdot)$ to denote the cost incurred. We claim that given a usage sequence $U$ such that $|U| \leq n^{*}$, OPT behaves as follows:

1. If $U$ is contiguous and $|U|=n^{*}$, then OPT will lease always or buy the equipment on the first period of the sequence. Notice that according to the definition of $n^{*}$, for 
such a sequence

$$
C(\operatorname{BUY}(\infty))=C(\operatorname{BUY}(1)) .
$$

That is, the cost of purchasing on the first usage period is the same as the cost of leasing throughout $U$.

2. If $|U|<n^{*}$, OPT always leases.

3. If $|U|=n^{*}$ is noncontiguous, then OPT always leases.

To prove this claim notice that if $0 \leq k<n \leq n^{*}$, then

$$
\begin{aligned}
C(\mathrm{BUY}(k)) & \geq C(\mathrm{BUY}(\infty)) \\
& \mathbb{1} \\
c \sum_{j=1}^{k} \beta^{u_{j}-1}+P \beta^{u_{k+1}-1} & \geq c \sum_{j=1}^{n} \beta^{u_{j}-1} \\
& \text { 业 } \\
& \geq c \sum_{j=k+1}^{n} \beta^{u_{j}-1} \\
& \mathbb{1} \beta^{u_{k+1}-1} \\
P & \geq c \sum_{j=k+1}^{n} \beta^{u_{j}-u_{k+1}},
\end{aligned}
$$

but

$$
c \sum_{j=k+1}^{n} \beta^{u_{j}-u_{k+1}} \leq c \sum_{j=1}^{n^{*}} \beta^{j}=P
$$

and equality holds in (4) if and only if $k=0$ and, for all $j, u_{j}=j$; that is, if and only if $U$ is contiguous with $|U|=n^{*}$. This proves the claim.

For a given noncontiguous time usage sequence $U$, let $\tilde{U}$ be obtained from $U$ by eliminating the last (nonusage) gap. When the on-line player applies $S^{* *}$, for every noncontiguous time usage sequence $U$ with $|U| \leq n^{*}, R(\tilde{U}) \geq R(U)$.

Assume, without loss of generality, that $|U|=n^{*}$. (If $|U|<n^{*}$, then both the on-line player and OPT always lease, therefore $R(\tilde{U})=R(U)$.)

Consider the last gap in the time usage sequence $U$ that occurs between $u_{k}$ and $u_{k+1}$, and assume that this gap lasts $l+1$ periods. The cost ratio achieved for the sequence $U$ is

$$
\begin{aligned}
R(U) & =\frac{c \sum_{i=1}^{n^{*}-1} \beta^{u_{i}-1}+P \beta^{u_{n^{*}-1}}}{c \sum_{i=1}^{n^{*}} \beta^{u_{i}-1}} \\
& =\frac{c \sum_{i=1}^{k} \beta^{u_{i}-1}+c \sum_{i=k+1}^{n^{*}-1} \beta^{u_{i}-1}+P \beta^{u_{n^{*}-1}}}{c \sum_{i=1}^{k} \beta^{u_{i}-1}+c \sum_{i=k+1}^{n^{*}-1} \beta^{u_{i}-1}+c \beta^{u_{n^{*}-1}}}
\end{aligned}
$$




$$
=\frac{c \sum_{i=1}^{k} \beta^{u_{i}-1}+c \beta^{l} \sum_{i=k+1}^{n^{*}-1} \beta^{u_{i}-1-l}+P \beta^{l} \beta^{u_{n^{*}-1-l}}}{c \sum_{i=1}^{k} \beta^{u_{i}-1}+c \beta^{l} \sum_{i=k+1}^{n^{*}-1} \beta^{u_{i}-1-l}+c \beta^{l} \beta^{u_{n^{*}-1-l}}} .
$$

Similarly, the cost ratio achieved for the sequence $\tilde{U}$ is

$$
R(\tilde{U})=\frac{c \sum_{i=1}^{i=k} \beta^{u_{i}-1}+c \sum_{i=k+1}^{i=n^{*}-1} \beta^{u_{i}-1-l}+P \beta^{u_{n^{*}-1-l}}}{c \sum_{i=1}^{i=k} \beta^{u_{i}-1}+c \sum_{i=k+1}^{i=n^{*}-1} \beta^{u_{i}-1-l}+c \beta^{u_{n^{*}-1-l}}} .
$$

Define

$$
\begin{aligned}
& X \stackrel{\text { def }}{=} c \sum_{i=1}^{i=k} \beta^{u_{i}-1} ; \\
& M \stackrel{\text { def }}{=} c \sum_{i=k+1}^{i=n^{*}-1} \beta^{u_{i}-1-l} .
\end{aligned}
$$

To complete the proof it remains to show that

$$
\begin{aligned}
& R(\tilde{U})=\frac{X+M+P \beta^{u_{n^{*}-1-l}}}{X+M+c \beta^{u_{n^{*}-1-l}}}>\frac{X+\beta^{l} M+P \beta^{l} \beta^{u_{n^{*}-1-l}}}{X+\beta^{l} M+c \beta^{l} \beta^{u_{n^{*}-1-l}}}=R(U) \\
& \text { 企 } \\
& 1+\frac{\beta^{u_{n^{*}-1-l}}(P-c)}{X+M+c \beta^{u_{n^{*}-1-l}}}>1+\frac{\beta^{l} \beta^{u_{n^{*}-1-l}}(P-c)}{X+\beta^{l} M+c \beta^{l} \beta^{u_{n^{*}-1-l}}} \\
& \text { 企 } \\
& \frac{1}{X+M+c \beta^{u_{n^{*}-1-l}}}>\frac{1}{X / \beta^{l}+M+c \beta^{u_{n^{*}-1-l}}} \text {. }
\end{aligned}
$$

Since $0<\beta<1$ and $l>0$ we get that the left-hand side in (5) is strictly greater than the right-hand side.

5. Playing Against Nature. Consider the following adversary that can choose any probability distribution $\pi$ on integers and then must announce it to the on-line player. The on-line player then chooses a strategy and an integer $n$ is sampled according to $\pi$. The leasing game is then played for $n$ periods without revealing $n$ to the on-line player until the $n$th period. We call such an adversary NATURE. At the outset it appears that NATURE is considerably weaker than the oblivious adversary and it could be concluded that by weakening the adversary we can hope for a better competitive ratio.

Somewhat surprisingly (see Remark 4) we have

THEOREM 5.1. In a game against NATURE the optimal competitive ratio is the same as in playing against an oblivious adversary.

PROOF. Consider the following two-person zero-sum game between the adversary and 
the on-line player:

- The set of pure strategies for the on-line player is $N-\{0\}$, where $N$ is the natural numbers and the pure strategy $t$ represents $S(t)$ (i.e., lease $t-1$ periods and then buy on the $t$ th period).

- The set of pure strategies for the adversary is $N-\{0\}$ where the pure strategy $n$ represents a decision to terminate the game on the $n$th period.

- The on-line player pays to the adversary according to the payment matrix $A_{t, n}=$ $R\left(t, n, n^{*}\right)$, in particular, if $i=0$, then $A_{t, n}=R(t, n, k)$.

- A mixed strategy $y$ for the on-line player is a probability distribution over $N-\{0\}$.

- A mixed strategy $x$ for the adversary is a probability distribution over $N-\{0\}$.

In the above game NATURE's objective is to ensure the value $\max _{x} \min _{y} y^{T} A x$ (which is the competitive ratio that can obtained in a game against NATURE).

In our analysis of the game against the oblivious adversary (Section 3.3) the optimal on-line strategy was designed to guarantee for the on-line player the value

$$
\min _{y} \max _{x} y^{T} A x
$$

Since for all $f, \inf _{y} \sup _{x} f(x, y) \geq \sup _{x} \inf _{y} f(x, y)$, it is only required to show that

$$
\max _{x} \min _{y} y^{T} A x \geq \min _{y} \max _{x} y^{T} A x .
$$

REMARK 4. Note that if the matrix $A$ was finite we could easily complete the proof by relying on the min-max theorem of game theory. However, in general the min-max theorem does not apply to infinite matrices (see, e.g., [2]).

We now prove inequality (6). We also characterize the set of probability distributions from which NATURE can choose in order to secure the highest possible competitive ratio.

Here we consider only the case $i=0$ (no interest rate) ${ }^{14}$ Denote the $j$ th row of $A$ by $A^{j}$. The payment matrix $A$ of the game is depicted in Figure 3.

From Theorem 3.2 we deduce that the value of $\min _{y} \max _{x} y^{T} A x$ is

$$
\alpha=2-\frac{(k /(k-1))^{k}-2}{(k /(k-1))^{k}-1} .
$$

From Lemma 3.1 we conclude that the optimal on-line randomized strategy has the property that, for all $j>k, y_{j}=0$, and from Lemma 3.3 we obtain that the optimal on-line randomized strategy has the property that, for all $j \leq k, y_{j} \neq 0$. Therefore, using the fact that $x$ is a probability distribution and the theorem of complementary slackness of linear programming (see, e.g., [2]) it must be that $\max _{x} \min _{y} y^{T} A x \geq \alpha$ if and only if the following set of restrictions is feasible:

- $\sum_{i=1}^{\infty} x_{i}=1$.

\footnotetext{
14 The proof of inequality (6) for the case $i>0$ is similar.
} 


\begin{tabular}{|c|c|c|c|c|c|c|c|c|c|c|c|}
\hline & $x_{1}$ & $x_{2}$ & $x_{3}$ & $\cdots$ & $x_{i}$ & $\ldots$ & $x_{k}$ & $x_{k \mid 1}$ & $x_{k+2}$ & $x_{k+3}$ & $\ldots$ \\
\hline$y_{1}$ & $\frac{P}{c}$ & $\frac{P}{2 c}$ & $\frac{p}{3 c}$ & $\cdots$ & $\frac{P}{j c}$ & $\ldots$ & $\frac{P}{k r}=1$ & 1 & 1 & 1 & $\cdots$ \\
\hline$y_{2}$ & 1 & $\frac{c+P}{2 c}$ & $\frac{c+P}{3 c}$ & $\cdots$ & $\frac{c+P}{j c}$ & $\cdots$ & $\frac{c+P}{k c}=1+\frac{1}{k}$ & $1+\frac{1}{k}$ & $1+\frac{1}{k}$ & $1+\frac{1}{k}$ & $\ldots$ \\
\hline$y_{3}$ & 1 & 1 & $\frac{2 c+Y}{3 c}$ & $\ldots$ & $\frac{2 c+P}{j c}$ & $\cdots$ & $\frac{2 c+P}{k c}=1+\frac{z}{k}$ & $1+\frac{2}{k}$ & $1+\frac{2}{k}$ & $1+\frac{2}{k}$ & $\ldots$ \\
\hline$\vdots$ & $\vdots$ & $\vdots$ & $\vdots$ & $\vdots$ & $\vdots$ & $\vdots$ & $\vdots$ & $\vdots$ & $\vdots$ & $\vdots$ & $\vdots$ \\
\hline$y_{j}$ & 1 & 1 & 1 & $\ldots$ & $\frac{(j-1) c+P}{j c}$ & $\ldots$ & $\frac{(j-1) c+P}{k c}=1+\frac{j-1}{k}$ & $1+\frac{j-1}{b}$ & $1+\frac{j-1}{k}$ & $1+\frac{j-1}{k}$ & $\ldots$ \\
\hline$\vdots$ & $\vdots$ & $\vdots$ & $\vdots$ & $\vdots$ & $\vdots$ & $\vdots$ & $\vdots$ & $\vdots$ & $\vdots$ & $\vdots$ & $\vdots$ \\
\hline$y_{k}$ & 1 & 1 & 1 & $\ldots$ & 1 & - & $\frac{(k-1) c+P}{k r}=1+\frac{k-1}{k}$ & $1+\frac{k-1}{k}$ & $1+\underset{k}{k-1}$ & $1 \mid \frac{k-1}{k}$ & ... \\
\hline$y_{k+1}$ & 1 & 1 & 1 & & 1 & .. & 1 & $\underset{k c}{k c+P}=2$ & 2 & 2 & $\cdots$ \\
\hline$y / k_{1} \mid 2$ & 1 & 1 & 1 & $\ldots$ & 1 & .. & 1 & $1+\frac{1}{k}$ & $2+\frac{1}{k}$ & $2+\frac{1}{k}$ & .. \\
\hline$y_{k+3}$ & 1 & 1 & 1 & $\ldots$ & 1 & $\cdots$ & 1 & $1+\frac{1}{k}$ & $1+\frac{2}{k}$ & $2+\frac{2}{k}$ & $\ldots$ \\
\hline$\vdots$ & $\vdots$ & $\vdots$ & $\vdots$ & $\vdots$ & $\vdots$ & $\vdots$ & $\vdots$ & $\vdots$ & $\vdots$ & $\vdots$ & $\vdots$ \\
\hline
\end{tabular}

Fig. 3. The payment matrix $A$ in a game against NATURE (when $i=0$ ).

- $\forall j \leq k, A^{j} \cdot x=\alpha$.

- $\forall j>k, A^{j} \cdot x \geq \alpha$.

By solving the equalities we obtain that,

$$
\forall 1 \leq j \leq k-1, \quad x_{j}=j\left(\frac{k-1}{k}\right)^{j-1} x_{1}=j \frac{\alpha}{k^{2}}\left(\frac{k-1}{k}\right)^{j-1} .
$$

Substituting $1 /\left(1-((k-1) / k)^{k}\right)$ for $\alpha$ we have,

$$
\forall 1 \leq j \leq k-1, \quad x_{j}=\frac{j}{k^{2}} \cdot \frac{((k-1) / k)^{j-1}}{1-((k-1) / k)^{k}} .
$$


LeMmA 5.1. Denote by $\gamma=\sum_{j=k}^{\infty} x_{j}=1-\sum_{i=1}^{k-1} x_{i}$. Then $x_{k}, x_{k+1}, \ldots, x_{k+m}, \ldots$ are characterized by:

- $\sum_{j=k}^{\infty} x_{j}=\gamma$.

- $\forall j \geq 0, x_{k+j} \leq((j+1) / k) \gamma-\sum_{i=0}^{j-1}(1+(j-i) / k) x_{k+i}$.

PROOF. Consider the following subset of restrictions:

$$
\forall j>k, \quad A^{j} \cdot x \geq \alpha .
$$

These restrictions translate into the following set of linear equations:

$$
\begin{aligned}
& \int \sum_{j=1}^{k} x_{j} \quad+2 \sum_{j=k+1}^{\infty} x_{j} \geq \alpha \\
& \sum_{j=1}^{k} x_{j}+\left(1+\frac{1}{k}\right) x_{k+1} \quad+\left(2+\frac{1}{k}\right) \sum_{j=k+2}^{\infty} x_{j} \geq \alpha, \\
& \left\{\sum_{j=1}^{k} x_{j}+\left(1+\frac{1}{k}\right) x_{k+1}+\left(1+\frac{2}{k}\right) x_{k+2}+\left(2+\frac{2}{k}\right) \sum_{j=k+3}^{\infty} x_{j} \quad \geq \alpha,\right. \\
& \sum_{j=1}^{k} x_{j}+\sum_{i=1}^{m-1}\left(1+\frac{i}{k}\right) x_{k+i} \quad+\left(2+\frac{m-1}{k}\right) \sum_{j=k+m}^{\infty} x_{j} \geq \alpha,
\end{aligned}
$$

Rearranging terms,

$$
\left\{\begin{array}{c}
\sum_{j=1}^{k} x_{j} \\
\sum_{j=1}^{k} x_{j}-x_{k+1} \\
\sum_{j=1}^{k} x_{j}-\left(1+\frac{1}{k}\right) x_{k+1} \\
\vdots \\
\sum_{j=1}^{k} x_{j}-\sum_{i=1}^{m-1}\left(1+\frac{m-1-i}{k}\right) x_{k+i} \\
\vdots
\end{array}\right.
$$$$
\begin{array}{ll}
+2 \sum_{j=k+1}^{\infty} x_{j} & \geq \alpha, \\
+\left(2+\frac{1}{k}\right) \sum_{j=k+1}^{\infty} x_{j} & \geq \alpha
\end{array}
$$$$
-x_{k+2}+\left(2+\frac{2}{k}\right) \sum_{j=k+1}^{\infty} x_{j} \quad \geq \alpha,
$$

By subtracting from each inequality the equation $A^{k} \cdot x=\alpha$ (written as $\sum_{j=1}^{k} x_{j}+$ $\left.(1-1 / k) x_{k}+(2-1 / k) \sum_{j=k+1}^{\infty} x_{j}=\alpha\right)$ and after straightforward manipulations we 


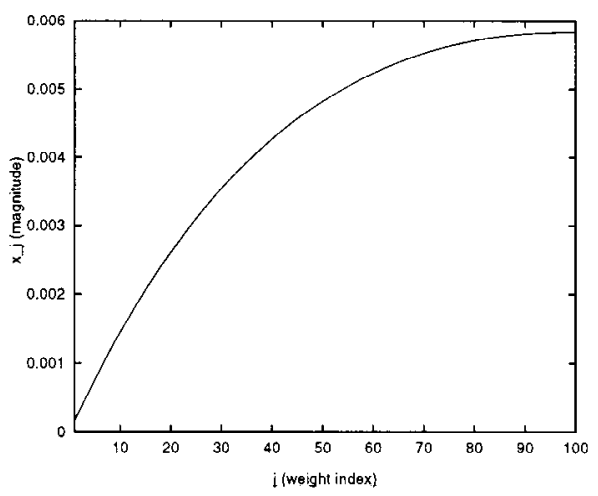

Fig. 4. The magnitude of the weights $x_{j}, 1 \leq j \leq k$, for $k=100$.

obtain

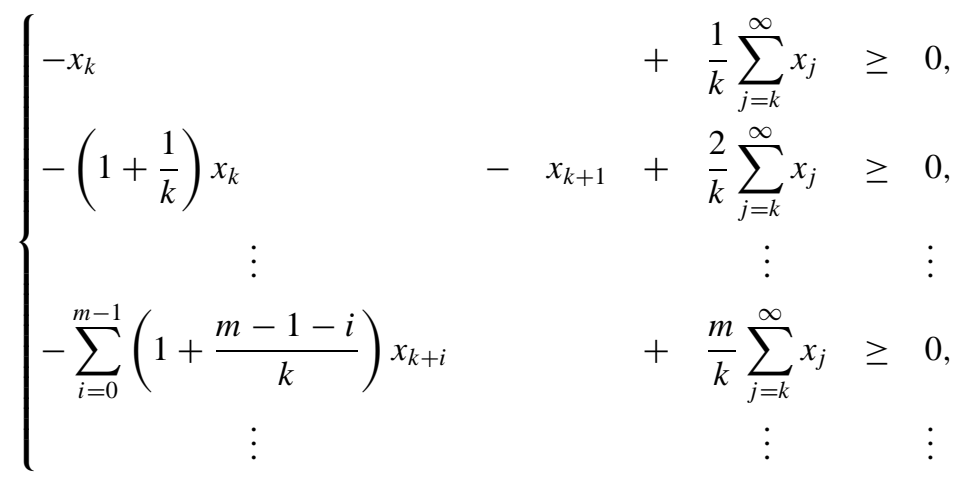

Clearly, there is a continuum of solutions of the inequalities in Lemma 5.1. Here we present one solution in which all the inequalities hold as equalities. Consider the set $\left\{x_{k+j}=((k-1) / k)^{j}(\gamma / k)\right\}_{j \geq 0}$. It can be verified (for example, by substitution) that this set specifies a solution in which all inequalities hold as equalities.

An illustration of the magnitude of the weights $x_{j}, 1 \leq j \leq k$, for $k=100$ is given in Figure 4.

LEMMA 5.2. In a game against NATURE, for all $k \geq 2$,

$$
P[\text { the game lasts at least } k \text { periods }]>\frac{1}{e-1} .
$$

PROOF.

$$
\sum_{j=1}^{k-1} x_{j}=\sum_{j=1}^{k-1} \frac{j}{k^{2}} \cdot \frac{((k-1) / k)^{j-1}}{\left(1-((k-1) / k)^{k}\right)}
$$




$$
\begin{aligned}
& =\frac{1}{k^{2}\left(1-((k-1) / k)^{k}\right)} \sum_{j=1}^{k-1} j\left(\frac{k-1}{k}\right)^{j-1} \\
& =\frac{1}{k^{2}\left(1-((k-1) / k)^{k}\right)}\left(\frac{k((k-1) / k)^{k-1}((k-1) / k-1)-((k-1) / k)^{k}+1}{((k-1) / k-1)^{2}}\right) \\
& =1-\frac{((k-1) / k)^{k-1}}{1-((k-1) / k)^{k}} .
\end{aligned}
$$

Since, for all $k \geq 2,((k-1) / k)^{k-1}>1 / e>((k-1) / k)^{k}$, it follows that

$$
1-\frac{((k-1) / k)^{k-1}}{1-((k-1) / k)^{k}}<1-\frac{1 / e}{1-1 / e}=1-\frac{1}{e-1} .
$$

Thus, there is an upper bound $(1-1 /(e-1))$ for the probability that the game will last less than $k$ periods. An intuitive explanation of this fact is that if with very high probability the game lasts less than $k$ periods, the on-line player will always lease and will attain a competitive ratio close to 1 . Therefore, if the adversary wants to enforce a high competitive ratio it must threaten the on-line player with a potentially far horizon.

6. Concluding Remarks. We have employed competitive analysis to study the leasing problem in a market with interest rates. Unlike most conventional approaches in finance we do not require the decision maker to have any prior knowledge about how long he will need the underlying asset for. Still however we show that by using a simple randomized algorithm the on-line player can achieve fairly low competitive ratios.

As discussed in Section 1.1 the pure competitive analysis (and our analyses here) blatantly ignore partial (probabilistic) knowledge that the on-line player may have. The risk-reward framework of al-Binali [7] that generalizes pure competitive analysis facilitates analyses and strategies that can include such side information. Indeed, in [7] al-Binali analyzes the folk variant of the leasing problem to exemplify the risk-reward framework. It would be very interesting to generalize our results to include risk management.

The basic leasing game studied here provides a reasonable abstraction of the leaseor-buy dilemma in a situation where the lease contract can be broken at any time with no penalty. Nevertheless, this basic formulation also abstracts away some realistic options that the lessee may have. For example, in practice after the equipment is bought it can later be sold (typically for a smaller price). Furthermore, our simple model ignores price uncertainty which is a considerable factor in any financial decision process. Our work can be extended in various directions:

- Introduce price uncertainty. In our model it is assumed that both the rental cost and the purchase cost are constants. The next step should be to account for price uncertainty, an important factor in financial modeling.

- Extend the analysis to a wider set of lease contracts. As noted in [31] some of the provisions that can be written into a lease are: (1) leases which grant the lessee the right to purchase the leases asset at a fixed price at the maturity date of the contract; 
(2) leases that are noncancelable during the life of the contract (this type of lease is called a financial lease); (3) leases that specify a period over which the lease is noncancelable followed by a period over which the lease can be canceled at any time.

- Refinement of the leasing problem by considering other economic factors such as inflation, taxes, salvage cost, etc.

\section{References}

[1] A. Abel. Asset prices under habit formation and catching up with the Joneses. American Economic Review, 80:43-47, May 1990.

[2] E. J. Anderson and P. Nash. Linear Programming in Infinite Dimentions. Wiley, Chichester, 1987.

[3] P. Auer, N. Cesa-Bianchi, Y. Freund, and R.E. Schapire. Gambling in a rigged casino: the adversarial multi-armed bandit problem. In Proceedings of the 36th Annual Symposium on Foundations of Computer Science, pages 322-331, 1995.

[4] B. Awerbuch, Y. Azar, A. Fiat, and T. Leighton. Making commitments in the face of uncertainty: how to pick a winner almost every time. In Proceedings of the 28th Annual ACM Symposium on Theory of Computing, 1996.

[5] Y. Azar, Y. Bartal, E. Feuerstein, A. Fiat, S. Leonardi, and A. Rosén. On capital investment. In Proceedings of 23rd ICALP, pages 429-441, 1996.

[6] S. Ben-David, A. Borodin, R. Karp, G. Tardos, and A. Widgerson. On the power of randomization in on-line algorithms. In Proceedings of the 22nd Symposium on Theory of Algorithms, pages 379-386, 1990.

[7] S. al-Binali. The competitive analysis of risk taking with application to online trading. In Proceedings of the 38th Annual Symposium on Foundations of Computer Science, 1997.

[8] A. Blum and A. Kalai. Universal portfolios with and without transaction costs. In Proceedings of the Tenth Annual Conference on Computational Learning Theory, 1997.

[9] A. Borodin and R. El-Yaniv. On randomization in online computation. In Proceedings of the Twelfth Annual IEEE Conference on Computational Complexity, pages 226-238, June 1997.

[10] A. Borodin, N. Linial, and M. Saks. An optimal online algorithm for metrical task systems. Journal of the ACM, 39:745-763, 1992.

[11] R.S. Bower. Issues in lease financing. Financial Management, 2:25-33, 1973.

[12] R. Brealey and S. Myers. Principles of Corporate Finance. McGraw-Hill, New York, 1996.

[13] A. Chou, J. Cooperstock, R. El-Yaniv, M. Klugerman, and T. Leighton. The statistical adversary allows optimal money-making trading strategies. In Proceedings of the 6th Annual ACM-SIAM Symposium on Discrete Algorithms, 1995.

[14] A. Chou, A. Shrivastava, and R. Sidney. On the power of magnitude. Unpublished manuscript, May 1995.

[15] T.M. Cover. Universal portfolios. Mathematical Finance, 1(1):1-29, January 1991.

[16] T.M. Cover and E. Ordentlich. Universal portfolios with side information. IEEE Transactions on Information Theory, March 1996.

[17] R. El-Yaniv, A. Fiat, R.M. Karp, and G. Turpin. Competitive analysis of financial games. In Proceedings of the 33rd Annual Symposium on Foundations of Computer Science, pages 327-333, 1992.

[18] R. El-Yaniv and R.M. Karp. Nearly optimal competitive online replacement policies. Mathematics of Operations Research, 22(4):814-839, 1997.

[19] J.R. Franks and S.D. Hodges. Valuation of financial lease contracts: a note. Journal of Finance, pages 657-660, May 1978.

[20] J. Gali. Keeping up with the Joneses: consumption externalities, portfolio choice, and asset prices. Journal of Money, Credit, and Banking, 26:1-8, February 1994.

[21] R.L. Graham. Bounds for certain multiprocessor anomalies. Bell Systems Technical Journal, 45:1531581, 1966.

[22] D.P. Helmbold, R.E. Schapire, Y. Singer, and M.K. Warmuth. On-line portfolio selecting using multiplicative updates, April 1996. To appear in Mathematical Finance.

[23] F. Jamshidian. Asymptotically optimal portfolios. Mathematical Finance, 2(2):131-150, 1992. 
[24] D.S. Johnson. Near-optimal bin packing algorithms. Ph.D. thesis, Massachusetts Institute of Technology, 1973.

[25] D.S. Johnson, A. Demers, J.D. Ullman, M.R. Garey, and R.L. Graham. Worst-case performance bounds for simple one-dimensional packing algorithms. SIAM Journal on Computing, 3:299-325, 1974.

[26] R.W. Johnson and W.G. Lewellen. Analysis of the lease-or-buy decision. Journal of Finance, 27:815824, September 1972.

[27] R. Kaniel. On the equipment rental problem. Master's thesis, Department of Computer Science, The Hebrew University of Jerusalem, July 1994.

[28] A. Karlin, M.S. Manasse, L.A. McGeoch, and S. Owicki. Competitive randomized algorithms for nonuniform problems. In Proceedings of the 1st ACM-SIAM Symposium on Discrete Algorithms, pages 300-309, 1990.

[29] A.R. Karlin, L. Rudolph, and D.D. Sleator. Competitive snoopy caching. Algorithmica, 3(1):70-119, 1988.

[30] R.M. Karp, R. Ostrovski, and Y. Rabani. 1993. Personal communication.

[31] J.J. McConnell and J.S. Schallheim. Valuation of asset leasing contracts. Journal of Financial Economics, 12:237-262, August 1983.

[32] M.H. Miller and C.W. Upton. Leasing, buying and the cost of capital services. Journal of Finance, 31:761-786, June 1976.

[33] S. Myers, D.A. Dill, and A.J. Bautista. Valuation of financial lease contracts. Journal of Finance, 31:799-820, June 1976.

[34] E. Ordentlich and T.M. Cover. On-line portfolio selection. In COLT 96, 1996.

[35] P. Raghavan. A statistical adversary for on-line algorithms. DIMACS Series in Discrete Mathematics and Theoretical Computer Science, volume 7, pages 79-83, 1992.

[36] L. Rudolph. Personal communication, 1994.

[37] L.D. Schall. The lease-or-buy and asset acquisition decisions. Journal of Finance, 29:1203-1214, September 1974.

[38] D. Sleator and R.E. Tarjan. Amortized efficiency of list update and paging rules. Communications of the ACM, 28:202-208, 1985.

[39] C.W. Smith Jr. and L.M. Wakeman. Determinations of corporate leasing policy. Journal of Finance, 40:895-910, July 1985.

[40] A.C. Yao. New algorithms for bin packing. Journal of the ACM, 27:207-227, 1980. 